\title{
Integrated Liquid Bismuth Propellant Feed System
}

\author{
Kurt A. Polzin, Thomas E. Markusic and Boris J. Stanojev \\ NASA-Marshall Space Flight Center \\ Huntsville, AL 35812
}

AIAA-2006-4636

\begin{abstract}
:
A prototype bismuth propellant feed and control system was constructed and tested. An electromagnetic pump was used in this system to provide fine control of the hydrostatic pressure, and a new type of in-line flow sensor was developed to provide an accurate, real-time measurement of the mass flow rate. High-temperature material compatibility was a driving design requirement for the pump and flow sensor, leading to the selection of macor for the main body of both components. Post-test inspections of both components revealed no cracks or leaking in either. In separate proof-of-concept experiments, the pump produced a linear pressure rise as a function of current that compared favorably with theoretical pump pressure predictions, with a pressure of $10 \mathrm{kPa}$ at $30 \mathrm{~A}$. Flow sensing was successfully demonstrated in a bench-top test using gallium as a substitute liquid metal. A real-time controller was successfully used to control the entire system, simultaneously monitoring all power supplies and performing data acquisition duties.
\end{abstract}




\title{
Integrated Liquid Bismuth Propellant Feed System
}

\author{
Kurt A. Polzin, ${ }^{*}$ Thomas E. Markusic, ${ }^{*}$ and Boris J. Stanojev ${ }^{\dagger}$ \\ NASA-Marshall Space Flight Center \\ Huntsville, AL 35812
}

AIAA-2006-4636

July $9-12,2006$

\begin{abstract}
A prototype bismuth propellant feed and control system was constructed and tested. An electromagnetic pump was used in this system to provide fine control of the hydrostatic pressure, and a new type of in-line flow sensor was developed to provide an accurate, real-time measurement of the mass flow rate. High-temperature material compatibility was a driving design requirement for the pump and flow sensor, leading to the selection of macor for the main body of both components. Post-test inspections of both components revealed no cracks or leaking in either. In separate proof-of-concept experiments, the pump produced a linear pressure rise as a function of current that compared favorably with theoretical pump pressure predictions, with a pressure of $10 \mathrm{kPa}$ at $30 \mathrm{~A}$. Flow sensing was successfully demonstrated in a bench-top test using gallium as a substitute liquid metal. A real-time controller was successfully used to control the entire system, simultaneously monitoring all power supplies and performing data acquisition duties.
\end{abstract}

\section{INTRODUCTION}

Operation of Hall thrusters with bismuth propellant has been shown to be a promising path for development of high-power (140 kW per thruster), highperformance $\left(8000 \mathrm{~s} I_{\mathrm{sp}}\right.$ at $>70 \%$ efficiency) electric propulsion systems[1]. The use of bismuth also alleviates several logistical issues that would normally be associated with development and deployment of a high-power Hall thruster operating on xenon, which is the traditional propellant option. The cost of propellant for testing and performance of deep-space missions is not nearly as prohibitive since bismuth costs far less than xenon $(\$ 75 / \mathrm{kg}$ compared to $\$ 2000 / \mathrm{kg}$ ). Also, since it is a solid at room temperature, vaporized bismuth can be condensed using simple, water-cooled plates, essentially 'cryopumping' the propellant at room-temperature and obtaining equivalent pumping speeds of millions of liters/sec. Finally, while Hall thrusters operating on xenon and other Noble gases have difficulty operating at high-voltages $(>1 \mathrm{kV})$, thrusters using bismuth have achieved very high $I_{\mathrm{sp}}$ because they can operate at voltages approaching $10 \mathrm{kV}$.

Presently, there are several efforts underway at various Iocations that aim to validate the high performance of bismuth-fed Hall thrusters and understand the physical mechanisms that allow for high-voltage, high-power, highperformance operation[2-4]. The work described in this

\footnotetext{
* Member AlAA.

†Senior Research Engineer, Madison Research Corp.

¥Presented at the $42^{s t}$ AIAA/ASME/SAE/ASEE Joint Propulsion Conference and Exhibit, Sacramento, CA. This material is declared a work of the U.S. Government and is not subject to copyright protection in the United States.
}

paper is part of the Very High $I_{\text {sp }}$ Thruster with Anode Layer (VHITAL) program[2]. Our effort was focused on the design and construction of a propellant management system that could deliver liquid bismuth to a Hall thruster while simultaneously monitoring the propellant flow rate. This is a critical element of the VHITAL program since performance cannot be accurately assessed without precise knowledge of the mass flow rate. Previous performance measurements[1] used a pre/post-test propellant weighing scheme that did not provide any real-time measurement of the mass flow rate during thruster operation, leading to relatively high error bars on both $I_{\mathrm{sp}}$ and thrust efficiency. The VHITAL propellant management system was designed to obtain more accurate, temporally resolved flow rate measurements. The overall system also served as a test bed where hardware and control algorithms could be evaluated for future propellant feed system development efforts.

In the next section, we define the problem while paying special attention to bismuth-specific issues that complicate our efforts. The feed system hardware that is actually in contact with molten bismuth is described in Sect. III while the electronics that control and monitor the hardware are discussed in Sect. IV. Section V contains data showing the performance of the electromagnetic pump and flow sensor.

\section{DEFINITION OF THE PROBLEM}

In the VHITAL thruster, the bismuth propellant must undergo three phase transitions: solid to liquid, liquid to gas, and gas to plasma. The role of the propellant management system we assembled is to deliver liquid bismuth to the vaporizer while simultaneously monitoring the mass flow rate. Thus, the system must both melt the propellant and possess the means to transport it. 
In our preliminary analysis we identified the following bismuth-specific challenges associated with the development of the VHITAL propellant management system:

- The high density of bismuth $\left(\mathcal{O}\left(10^{4}\right) \mathrm{kg} / \mathrm{m}^{3}\right)$ and low propellant mass flow rate $\left(\mathcal{O}\left(10^{-5}\right) \mathrm{kg} / \mathrm{sec}\right)$ results in a very low volume flow rate $\left(\mathcal{O}\left(10^{-9}-10^{-10}\right)\right.$ $\mathrm{m}^{3} / \mathrm{sec}$ ) that is challenging to continuously monitor using in-line flow sensors. The high density of the propellant also causes gravitational influences on the feed-line pressure - as the propellant level in the tank recedes during testing - which must be compensated for by varying the pumping pressure.

- The high melting temperature of bismuth $(\sim 275$ ${ }^{\circ} \mathrm{C}$ ) makes makes it difficult to employ many "offthe-shelf" components (e.g., valves and electronics). Consequently, many of the feed system components require custom design and fabrication.

- The relatively low electrical conductivity of bismuth makes it nearly impossible to measure the flow rate electromagnetically as was previously done for lithium[5]. Flow sensing elements that are in direct contact with the flow must be electrically insulated, but the high temperature of molten bismuth precludes the use of some of the most attractive insulators. Finally, the feed system and any electrical connections must be shielded from condensing bismuth vapor (from the thruster exhaust) as this can electrically short some of the feed system components - rendering them inoperable.

\section{FEED SYSTEM HARDWARE}

The propellant management system we designed to tackle the difficulties listed in the previous section operates in the following manner. A propellant reservoir containing solid bismuth is heated until its temperature is above bismuth's melting temperature. The liquid bismuth can be made to move using a combination of two different techniques. The application of gas pressure to the reservoir forces molten bismuth through the system. The second method, which exploits the fact that bismuth is an electrically conducting fluid, employs an electromagnetic (EM) pump that is integrated into the propellant feed line downstream of the reservoir. An in-line flow sensor capable of making real-time measurements completes the propellant feed system. The entire system is shown in Fig. 1. In what follows, each of these four major components are discussed in greater detail.

\section{A. Propellant Reservoir}

The reservoir is designed to allow for the storage and melting of high purity bismuth. It is fabricated from $316 \mathrm{~L}$ stainless steel and has a removable lid for loading bismuth.

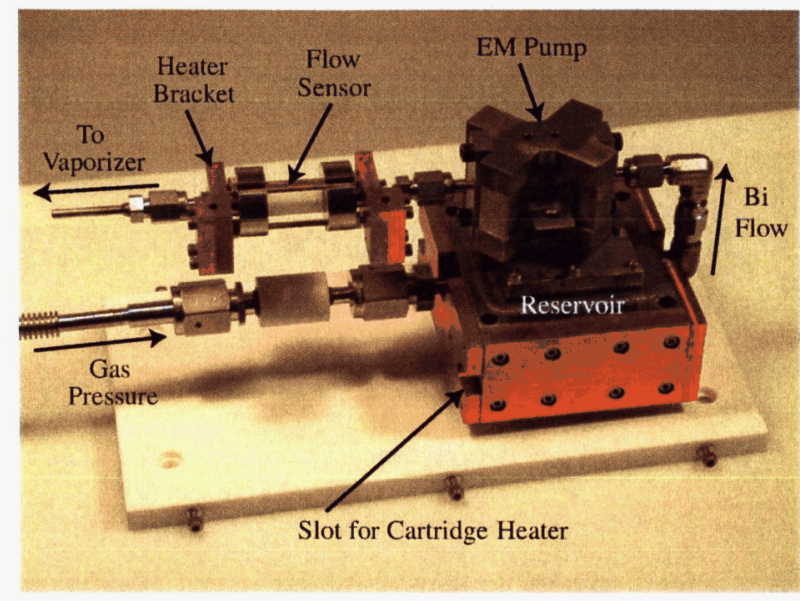

FIG. 1: Assembled bismuth propellant feed system.

An inlet tube welded into the body allows for gas pressurization that forces liquid bismuth out of the reservoir through an outlet tube. Two three-inch long, 1/4" diameter cartridge heaters are used to heat the reservoir. The copper plates bolted to the steel structure are employed to uniformly distribute heat to the reservoir.

\section{B. Gas Pressurization System}

The gas pressurization system (shown photographically in Fig. 2 and schematically in Fig. 3 ) is designed to provide gas pressurization to the reservoir within the (continuously adjustable) range of 0-200 Torr. The system is designed to operate inside a vacuum chamber, allowing for ease of integration with other thruster subsystems.

The pressure vessel (PV) is a 0.7 liter, fiber wound cylinder rated to $4500 \mathrm{psi}$. The hand-operated regulator, which reduces the high pressure in the reservoir to the lower pressure required for the electro-pneumatic regulator (EPR) actually limits the pressure rating of the system to roughly 200 psi. Two solenoid operated valves (SOV1 and SOV2) are used to isolate the propellant reservoir from the gas pressurization system and vent the pressurized lines directly to vacuum.

The EPR is the heart of the gas-pressurization system, using a "bang-bang" arrangement of solenoid valves. The (propellant reservoir) pressure is set to the desired value by alternately opening and closing the high pressure and vacuum valves. An on-board control system uses a feedback signal from an on-board pressure transducer to determine the proper sequencing of the SOV open/close operations. External control is maintained using a $0-10 \mathrm{VDC}$ signal that allows for remote adjustment of the reservoir gas pressure to the desired value. 


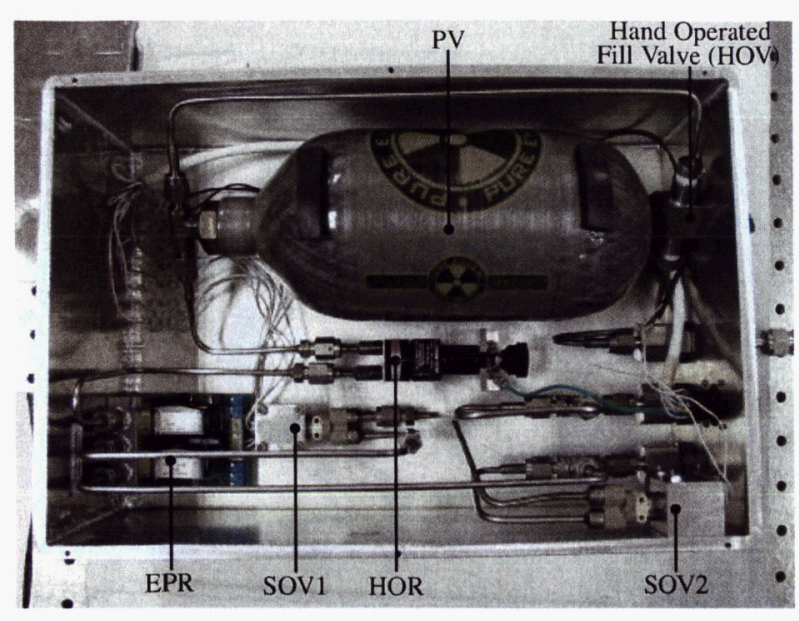

FIG. 2: Photograph of the gas pressurization system.

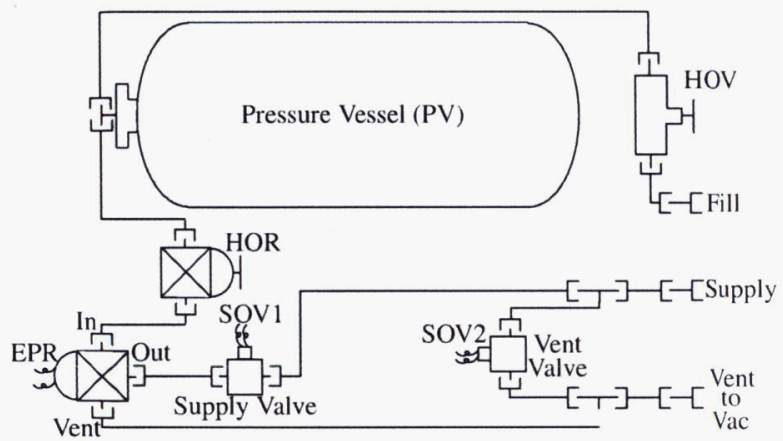

FIG. 3: Schematic representation of the gas pressurization system.

\section{Electromagnetic Pump}

For the sake of brevity, we refer the reader to Ref. [6] for a review of electromagnetic pump theory and a survey of the current state-of-the-art. We employed glass-mica ceramic (also known as macor) in the construction of the main body of our EM pump because it is stable and non-reactive with liquid bismuth near $300{ }^{\circ} \mathrm{C}$ temperature. It is also inexpensive and easily machinable when compared to other ceramics.

The ease of fabrication afforded by the use of macor (versus, for example, aluminum nitride) allowed us to pursue an aggressive design for the EM pump. Samarium cobalt magnets were employed, with a magnet separation of 2.9 $\mathrm{mm}$ and a channel height $s$ of $2.0 \mathrm{~mm}$. This resulted in an on-axis magnetic field strength $B$ of $0.64 \mathrm{~T}$ and gave $B / s=3.2 \mathrm{~T} / \mathrm{cm}$.

The major components of the bismuth EM pump are shown in Fig. 4. The CAD model is included to show a sectioned view that reveals the inner construction. The materials used to fabricate the pump were: macor (pump body), iron (magnet yoke), 316L stainless steel (end caps and feed lines), Inconnel (electrodes), and samarium cobalt (magnets). The electrodes were bonded to the macor body using high-temperature epoxy and the feed lines were welded to the end caps. The feed lines utilize Swagelok VCR fittings for attachment to the rest of the feed system and two Parker metal c-rings seal the joints between the end caps and the ceramic body. Two small (1" long, 1/8" diameter) cartridge heaters (not pictured) were used to heat the end caps and feed lines where they joined to the pump to ensure the bismuth would remain molten while it traversed through the pump.

\section{Flow Sensor}

We have developed a new type of flow sensor, which we call the 'hotspot' flow sensor[5]. The device operates in the manner illustrated in Fig. 5. A pulse of thermal energy (derived from a current pulse and associated joule heating) is applied near the inlet of the sensor. The flow is 'tagged' with a thermal feature that is convected downstream by the flowing liquid metal. A downstream thermocouple records a 'ripple' in the local temperature associated with the passing 'hotspot in the propellant. By measuring the time between the upstream generation and downstream detection of the thermal feature, the flow speed can be calculated using a time of flight analysis.

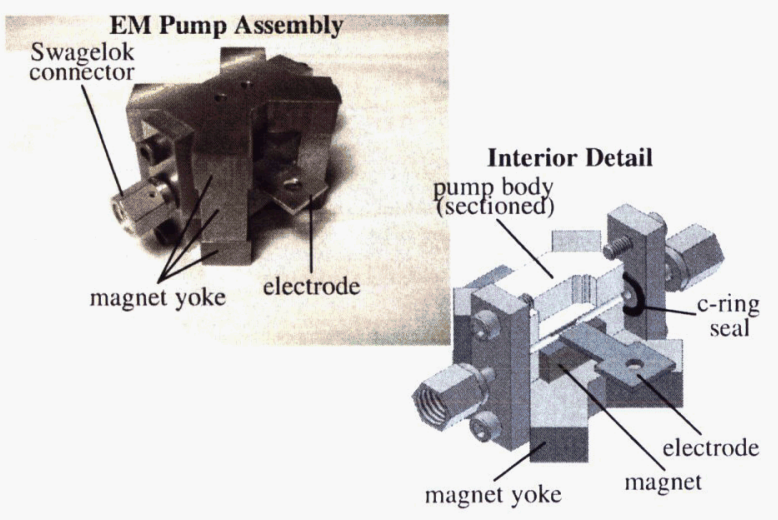

FIG. 4: Photograph of assembled EM pump and a CAD drawing sectioned to show the pump's inner detail.

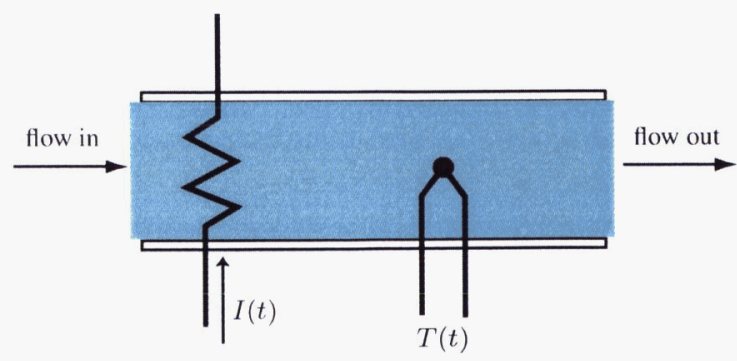

FIG. 5: Principle of operation of the hotspot flow sensor. 

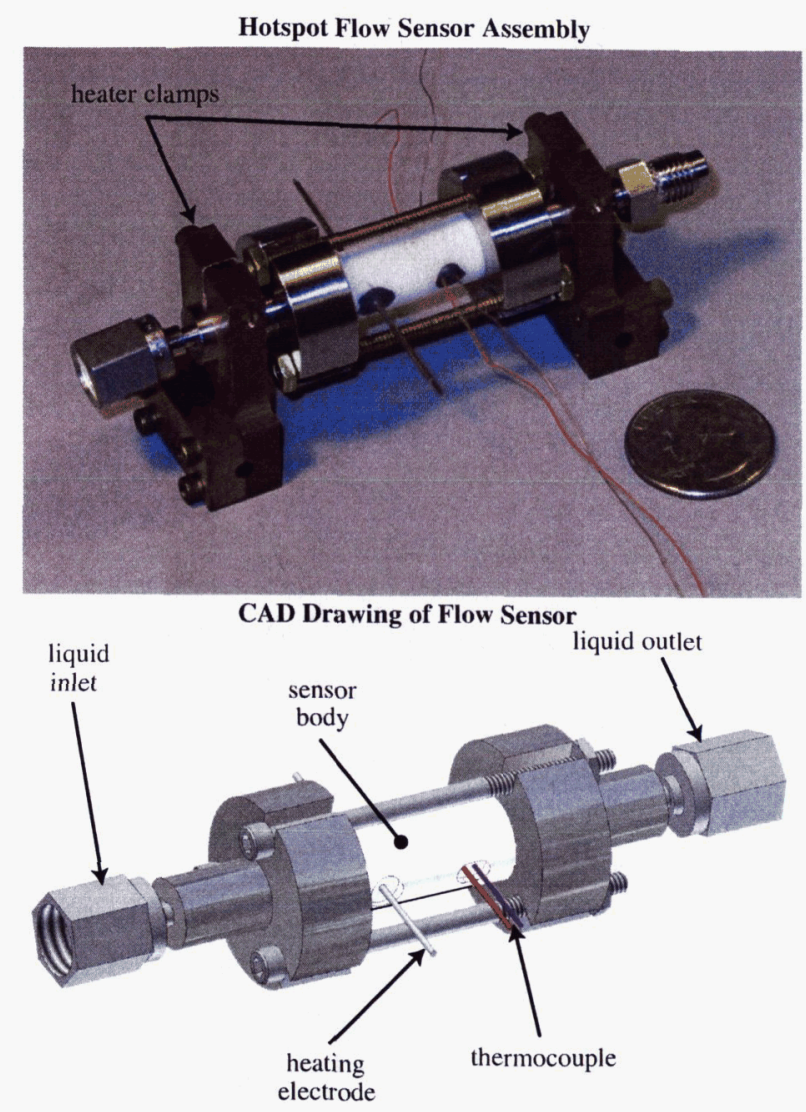

FIG. 6: Photograph and CAD drawing of assembled flow sensor.

The primary advantage of this technique is that it doesn't depend on an absolute measurement of temperature, but instead relies on the observation of thermal features. This makes the technique insensitive to other externally generated thermal fluctuations. The hotspot in the upstream flow is generated by pulsing current directly through the liquid metal; by doing so we exploit the intrinsic resistivity of the fluid and obviate the need for a separate resistive heating element. In order for the hotspot flow sensor to provide useful results, the spatial integrity of the hotspot must be maintained until it reaches the thermocouple location. The hotspot will tend to decrease in magnitude as it propagates, due to thermal diffusion. Therefore we have designed the device such that the thermal diffusion time scale is much longer than the convective time scale.

As with the pump, the flow sensor was constructed around a macor central body (see Fig. 6). The fluid connectors at the ends of the sensor and the heating electrodes were fabricated from $316 \mathrm{~L}$ stainless steel. The downstream temperature measurement was made using a 0.002 " diameter butt-welded type $\mathrm{E}$ thermocouple that penetrates the macor body, with approximately $1 \mathrm{~cm}$ separation from the heating location. The fluid connectors were sealed to the macor body using Parker metal c-rings while the electrodes and thermocouples were sealed using high tempera-

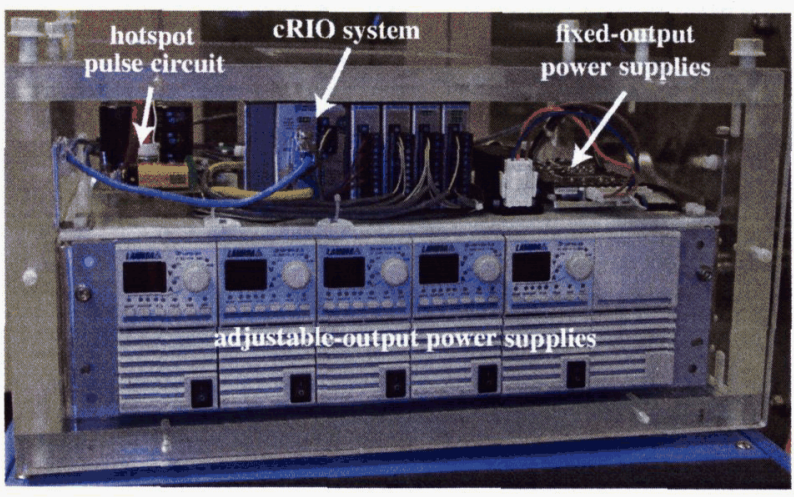

FIG. 7: Control system electronics suite for the VHITAL feed system.

ture epoxy. Heaters were clamped to the fluid connectors on the flow sensor to inhibit propellant freezing. The flow channel has a diameter of 0.022 ", which will resulted in a bismuth flow speed of $\sim 1 \mathrm{~cm} / \mathrm{sec}$ at a mass flow rate of $\sim 10 \mathrm{mg} / \mathrm{sec}$.

\section{CONTROL SYSTEM}

The VHITAL propellant management control system must not only control all the components described in the previous section, but must also perform all the data sampling tasks required during operation of the feed system. Tasks include operation, adjustment, and switching of the various power supplies connected to the experiment, monitoring of any analog voltages generated by components in the system, operation of the hotspot flow sensor circuitry, and performance of temperature measurements to monitor the overall state of the feed system. This last task is made more difficult by the need to sample the flow sensor thermocouple temperature as quickly as possible to achieve an accurate measure of the flow rate.

The electronics suite developed for the VHITAL feed system (see Fig. 7) is centered around a compact Reconfigurable I/O (cRIO) real-time embedded control system. This system performs all communications and data sampling required for feed system operation. The entire system has been tested in conjunction with the feed system hardware and operated within the expected parameters. We proceed first with a discussion of the embedded control system architecture and then describe the electronic subsystems that operate in conjunction with the embedded controller to form the complete control system.

\section{A. System Architecture}

In general, the embedded control system can be operated as either a stand-alone system or in conjunction with an external computer. The latter mode of operation is the one we 


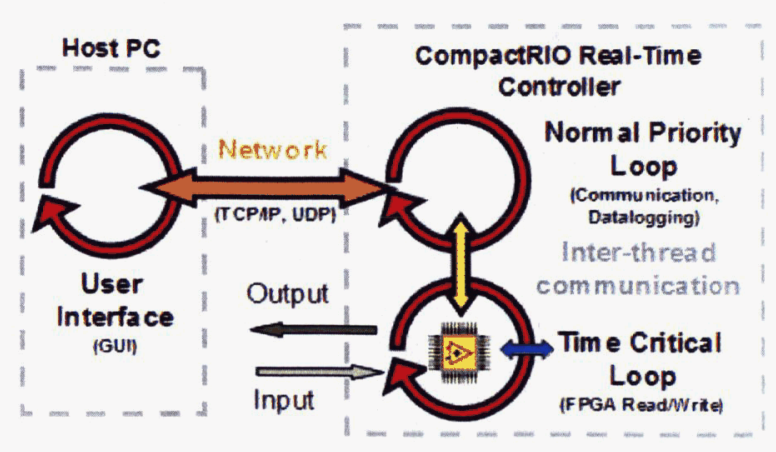

FIG. 8: Control system architecture and data flow (from ni.com).

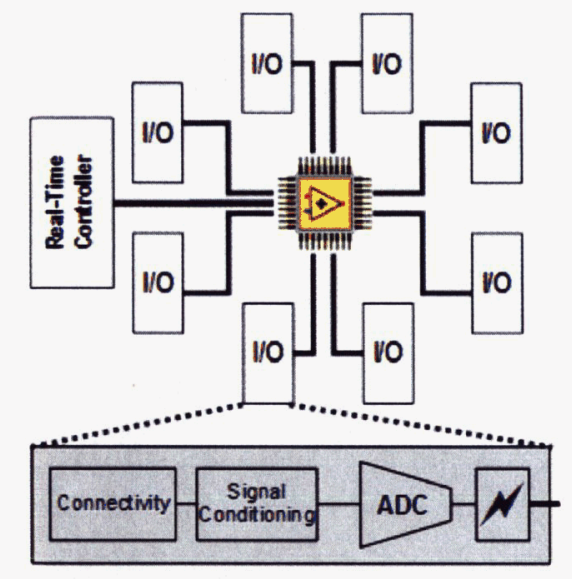

FIG. 9: cRIO real-time controller/FPGA architecture (from ni.com).

have implemented in the VHITAL system. In this mode, the embedded controller is passive and receives all commands from the external computer (in our case, connected using a fiber optic network converter for electrical isolation). The controller executes all the commands it receives and simultaneously monitors the feed system using if/else logic to prevent hardware damage. A schematic showing the top level control system architecture and the flow of command and data signals between the various components is shown in Fig. 8.

The user interface was designed in Labview and is used both to issue commands to the embedded controller and to display data from the control and data acquisition systems in real time. In principle, any standard computer with networking capability can operate as the user interface machine. The embedded controller hardware combines small format PC capabilities with a fast Field Programmable Gate Array (FPGA). The cRIO system is designed for rapid prototyping and fast signal conditioning operation using the FPGA clock for data acquisition. This system was selected for the VHITAL application because of the compact size of the cRIO unit and the various available I/O modules.

\section{B. The cRIO Chassis}

The cRIO chassis accommodates four modules, which can be selected depending on the numbers and types of $\mathrm{L} / \mathrm{Os}$ required for an application. Each module has between 4 and 32 channels, depending upon the function of the module. The following is a complete description of the cRIO modules we used:

- NI cRIO-9002: real-time controller with $32 \mathrm{MB}$ DRAM, 64 MB compact flash

- NI cRIO-9201: 8-channel, 10 V, 500 kS/s, 12-Bit analog input module

- NI cRIO-9481: 4-channel, sourcing digital output single-pole single-throw relay module

- NI cRIO-9472: 8-channel, 24 V Logic, 100 s, sourcing digital output module

- NI cRIO-9211: 4-channel, 14 sample/s, 24-Bit, 80 $\mathrm{mV}$ thermocouple input module

Each module connects to the real time controller through the FPGA module as shown on Fig. 9.

\section{Power Supplies}

The control system uses two separate power subsystems. A Vicor dual output AC/DC converter is used to provide power to the electronics. These include the cRIO module, optical network converter, and cooling fan. In addition, this supply is switched using the cRIO-9481 module to energize the solenoid valves SOV1 and SOV2 in the gas pressurization system. Five variable Lambda ZUP output power supplies are used to provide adjustable power to the feed system. These supplies were selected because they have a small form factor, are easily packaged into a cluster, and can be controlled through a serial connection. The supplies are interconnected and adjusted by the real-time controller using the RS-485 communication protocol. The supplies monitor their current and voltage outputs and send these data back to the controller using the same communications protocol. The following is a list of the specifications and primary function of each power supply:

- Magnetic pump - $6 \mathrm{~V} / 66 \mathrm{~A}$

- Component cartridge heaters - $80 \mathrm{~V} / 2.5 \mathrm{~A}$

- Reservoir cartridge heaters - 120 V/3.6 A

- Flow sensor capacitor charge - $120 \mathrm{~V} / 1.8 \mathrm{~A}$

- Gas pressure regulator control signal - 10 V/20 A 


\section{Flow Sensor Pulse Circuitry}

The 'hotspot' pulse circuitry is designed to store energy in capacitors and then release that energy in a fast pulse to thermally tag the liquid bismuth flowing between the electrodes in the flow sensor through Ohmic heating. Measurements of the temperature in the flow are obtained using a thermocouple connected to the cRIO-9211 module. The flow rate is calculated by taking the time difference between the initiation of the heating pulse and the detection of the temperature "peak" as it is convected downstream.

In the pulse circuit, an SCR is used to switch the energy from the capacitor bank into the flow sensor. The SCR is optically isolated from the control system through an optocoupler, thus minimizing the risk of exposing high current to the controller. The embedded controller initiates a current pulse by triggering the SCR gate through the optocoupler. To stop current from flowing, a serial command is sent to the power supply that charges the capacitor bank, setting it to output zero power. This drops the leakage current below the 'keep-alive' threshold of the SCR, allowing the solid-state switch to open. While this active shutdown provides total isolation between the charge circuit and the real-time controller, it is also a relatively slow process.

\section{COMPONENT PERFORMANCE}

The entire system was tested using hot $\left(>300^{\circ} \mathrm{C}\right)$, flowing bismuth. Once primed, the pump operated successfully and we could drive current between the electrodes in the hotspot sensor. To date, we have not been able to successfully acquire hotspot flow sensor data using bismuth as a propellant. Post inspection of the pump and flow sensor revealed no cracking of the macor bodies and no bismuth leaks at the seals. Additional tests meant to quantify the performance of the pump and flow sensor are detailed below.

\section{A. Pump Pressure}

The EM pump was previously evaluated[6] by measuring the hydrostatic pressure developed by the pump as a function of input current $I$. In order to eliminate the difficulties associated with handling liquid bismuth, these tests were conducted using a substitute liquid metal - gallium. The theoretical pressure $P$ developed by an EM pump is independent of particular properties of the conducting fluid and given by the equation

$$
P=\frac{B}{s} I
$$

where $B$ is the magnetic field strength in the channel and $s$ is the width of the conduction current path through the liquid metal.

Applied current levels ranging from 10-30 A were tested and the results are shown in Fig. 10. As expected, the data

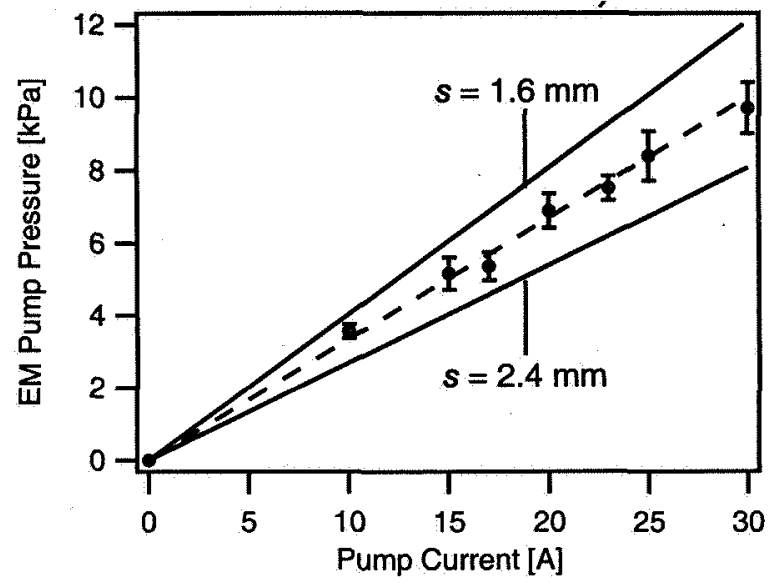

FIG. 10: EM pump pressure versus current (the solid lines are theoretical curves based on the value of $s$ given, the dashed line represents a linear curve fit of the data).

show that the pressure developed by the EM pump is linear with current. A linear curve fit (dashed line) yields a value for $B / s$ equal to $334 \pm 16 \mathrm{~Pa} / \mathrm{A}$. The process of comparing this to the predicted pump performance is complicated by the ambiguity in the definition of the channel height $s$. The pump was constructed by drilling an axial, $2.4 \mathrm{~mm}$ hole through which the liquid metal flows. However, the electrodes penetrate through the sides of the pump and are somewhat smaller in height $(1.6 \mathrm{~mm})$. The value of $s$ is dependent on the (unknown) current density distribution. However, the geometry of our EM pump allows us to bound the effective value of $s$ between $1.6-2.4 \mathrm{~mm}$. Theoretical lines for each of these extremes are shown in Fig. 10. The measured data show good quantitative agreement, lying roughly midway between the two theoretical curves.

The data demonstrate that the bismuth EM pump is capable of delivering the required hydrostatic pressure $\left(\mathcal{O}\left(10^{3}\right)\right.$ $\mathrm{Pa}$ ) for electric propulsion applications. Furthermore, the pump is seen to operate at a level consistent with the theoretical maximum performance. This implies that losses associated with stray conduction currents are negligible in the present design.

\section{B. Flow Sensor Measurements}

The current output as a function of time is plotted in Fig. 11. For the an initial capacitor charge of $15 \mathrm{~V}$, the pulse peaked just under $80 \mathrm{~A}$ and lasted roughly $1 \mathrm{~ms}$. While the magnitude of this output will change with the initial capacitor voltage, the waveforms will not change in shape.

Temperature measurements obtained downstream of the location where the heat pulse was introduced are presented in Fig. 12 for gallium, which was used for our initial proof-of-concept experiments because it is easier to handle (low temperature experimentation). Time is measured 


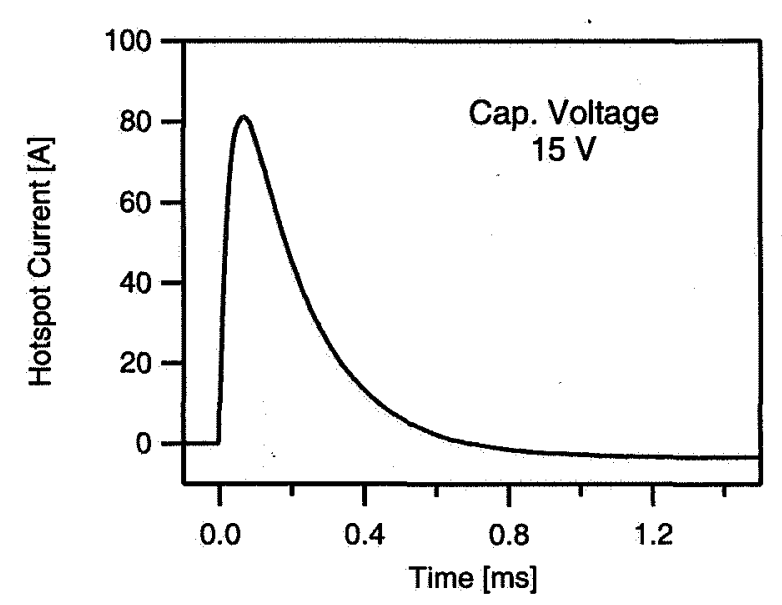

FIG. 11: Current output of the hotspot pulse circuit for an initial charge voltage of $15 \mathrm{~V}$.

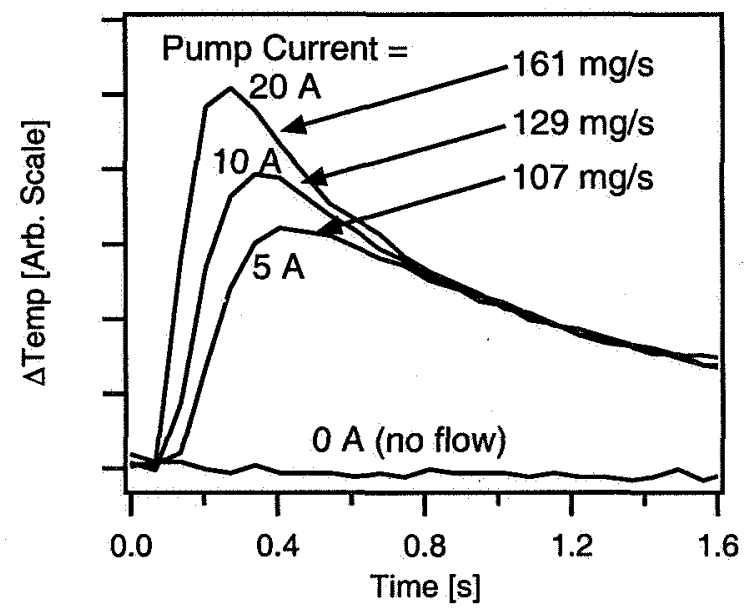

FIG. 12: Measured gallium fluid temperatures downstream of the current pulse location for different EM pump currents. Flow rates are estimated based upon the sensor geometry, fluid density and hotspot time-of-flight.

from when the current pulse is initiated. Several representative temperature waveforms are shown, corresponding to different EM pump operating currents (different flow rates). The data demonstrate that the peak of the thermal pulse passes the thermocouple location earlier in time as the EM pump current, and the associated fluid flow rate, is increased. Mass flow rates were estimated based upon the geometry of the flow sensor, the density of the fluid, and the heat pulse time-of-flight measurement. In these proofof-concept experiments we were able to demonstrate measurement of mass flow rates ranging from 0 to roughly 160 $\mathrm{mg} / \mathrm{s}$. The flow rates are much higher in this case than those expected in the VHITAL thruster because our flow loop did not have the added flow impedance of a vaporizer.

\section{SUMMARY AND CONCLUSIONS}

We have presented a design for a bismuth propellant management system that delivers liquid bismuth to a vaporizer while simultaneously monitoring the mass-flow rate in real time. This system has been assembled as a series of modules, allowing it to serve as a test bed for hardware and control algorithm development. We can draw the following conclusions from this development effort.

- High-temperature material compatibility was a driving design requirement for the bismuth $\mathrm{EM}$ pump and flow sensor. Macor was chosen for the insulating body material to minimize shunt conduction current losses. Metal c-rings were used to seal the the feed lines to the macor bodies. Tests with hot flowing bismuth successfully demonstrated operation of the pump and post test inspection revealed no cracking or leaks in either component.

- The EM pump produced a hydrodynamic pressure in the liquid metal of $10 \mathrm{kPa}$ when operating at $30 \mathrm{~A}$. A measure of pump pressure as a function of input current showed good quantitative agreement with the theoretical pump output.

- The flow sensing technique employed by the hotspot flow sensor was successfully demonstrated using gallium. Mass flow rates were estimated based upon the heat pulse time-of-flight and the geometry of the fluid channel and spanned a range from 0 to 160 $\mathrm{mg} / \mathrm{s}$.

- A cRIO real-time controller, coupled to a remote computer/user interface through a fiber optic network converter, was used to control all feed system electronics and simultaneously perform all data acquisition. We demonstrated operation of the entire system (power supplies, pulse circuit, data acquisition) and were able to control and monitor the feed system using our remote computer interface.

\section{Acknowledgments}

We appreciate the management support of Mike Fazah and Jim Martin and the program office support of Dr. Michael LaPointe throughout the duration of this effort. We also acknowledge the contributions of Doug Davenport, Doug Galloway, Tommy Reid, Keith Chavers, Rondal Boutwell and Jeff Gross. The VHITAL program was supported by NASAs Exploration Systems Mission Directorate (Project Prometheus) and funded under contract NAS7-03001 managed by John Warren. 
[1] S.O. Tverdokhlebov, A.V. Semenkin, and J.E. Polk. "Bismuth propellant option for very high power TAL thruster". 40th AIAA Aerospace Sciences Meeting, Jan. 14-17, 2002. AIAA Paper 2002-348.

[2] A. Sengupta, et al. "An overview of the VHITAL program: a two-stage bismuth fed very high specific impulse thruster with anode layer". 29th International Electric Propulsion Conference, Princeton, NJ, Oct. 31-Nov. 4, 2005. IEPC-2005238.

[3] J. Szabo, C. Gasdaska, V. Hruby, and M. Robin. "Bismuth Hall thruster with ceramic discharge channel". Proc. 53rd JANNAF Propulsion Meeting, Monterey, CA, Dec. 2005.

[4] A. Kieckhafer, D. Massey, and L.B. King. "Probe diagnos- tics in a bismuth Hall thruster". 29th International Electric Propulsion Conference, Princeton, NJ, Oct. 31-Nov. 4, 2005. IEPC-2005-129.

[5] T.E. Markusic, K.A. Polzin, B.J. Stanojev, C. Dodson, and A. Dehoyos. "Liquid metal flow sensors for electric propulsion". Proc. 53rd JANNAF Propulsion Meeting, Monterey, CA, Dec. 2005.

[6] T.E. Markusic, K.A. Polzin, and A. Dehoyos. "Electromagnetic pumps for conductive-propellant feed systems". 29th International Electric Propulsion Conference, Princeton, NJ, Oct. 31-Nov. 4, 2005. IEPC-2005-295. 